\title{
Analysis of Mobile Apps for Learning Grammar through Mobile Assisted Language Learning Approach
}

\author{
Ebrahim Panah \\ University College of Yayasan Pahang, \\ Pahang, Malaysia \\ https://orcid.org/0000-0003-1866-2750 \\ Som, Hafizan Mat \\ University College of Yayasan Pahang \\ Pahang, Malaysia \\ https://orcid.org/0000-0002-5282-6141 \\ Muhammad Yasir Babar \\ Thompson Rivers University, Canada \\ https:// orcid.org/0000-0001-5262-9933 \\ Sedigheh Shakib \\ Erican College \\ Kualaumpur, Malaysia \\ https:// orcid.org/0000-0001-6249-0293
}

\author{
Anahita Ghanad \\ Veritas University College \\ Kuala Lumpur, Malaysia \\ https:// orcid.org/0000-0002-6368-4126
}

\begin{tabular}{|c|c|c|}
\hline Received & Revised & Accepted \\
\hline 28 August 2021 & 28 October 2021 & 15 November 2021 \\
\hline
\end{tabular}

\begin{abstract}
The objective of the current study is to evaluate the recently developed mobile apps for learning and improving English grammar. This study selected 10 grammar apps ranked 3 and above by online users with a large number of installations. The study adapted a framework proposed by Supyan Hussin (2013) with four criteria: System, Program, Curriculum, and Language \& Culture. Furthermore, information provided in Google Play Store App assessment in terms of App users' Reviews, Ratings, and Downloads also was used to assess the selected grammar apps. First, the findings show that the grammar apps tend to teach grammar out of context, second, apps minimally adapt to the user's skill sets. Third, the grammar apps rarely offer explanatory corrective feedback to the users. Fourth, some grammar apps have accuracy issue in terms content and typo. Despite pedagogical paradigm shift to communicative approaches to language learning, the assessed grammar
\end{abstract}


apps are more behaviorists in nature where features of cognitivism and constructivism, and particularly connectivism approaches are rare. To better align with MALL, contextualized language, explanatory feedback, and adaptive technology need to be incorporated into these apps.

Keywords: Mobile apps; MALL; English Grammar

\section{Introduction}

Information technology has virtually removed the border among countries and global village has been created. It has affected all walks of human life including education. Smart phones as another technology have been around for more than two decades and their widespread usage coupled with their affordance and advancement in function has led to the development of numerous mobile apps for learning, particularly teaching language and learning grammar (RodríguezArancón, Arús, \& Calle-Martínez, 2013; Usagawa, 2018). Mobile devices offer learning opportunities such as informal, contextual, spontaneous, portable, pervasive, ubiquitous, and personal (Kukulska-Hulme et al., 2011). In using mobile apps for language learning, several elements such as teachers, learners, content, assessment and environment play important role in using mobile apps (Kim\& Kwon, 2012). Further, system, program, curriculum and language \& culture are important factors in selecting language apps (Supyan Hussin, 2013), which will be discussed in the subsequent section. Mobile apps make language learning more interesting and help learners develop their language skills positively. Both teachers and students have positive views and feedback to mobile applications (Eppard, Nasser, \& Reddy, 2016; Fabian, \& MacLean, 2014; Ganapathy, Shuib \& Azizan, 2016; Usagawa, 2018). Mobile apps support collaborative learning and self-pace learning (Kim \& Kwon, 2012). They provide interesting language learning environment where immediate feedback for students are provided and they can enhance learning performance ( $\mathrm{Wu}$ et al., 2012; Yunita, 2018). Hence, mobile app capabilities could be translated into huge opportunities for language teaching and learning (Rodríguez-Arancón, Arús, \& Calle-Martínez, 2013). However, some mobile apps have drawbacks, which need to be addressed (Wang et al., 2017). Mobile apps work based on three platforms, namely Android, Apple IOS and Microsoft windows 7 and have the potential to drastically change the field of language learning and teaching (RodríguezArancón, Arús, \& Calle-Martínez, 2013) as well as reshaping language learning styles and preferences (Kim \& Kwon, 2012).

Pilar, Jorge and Cristina (2013), based on quality criteria in Fernández-Pampillón et al., (2012), developed a rubric with some factors such as cognitive value \& pedagogic coherence, content quality, capacity to generate learning, interactivity \& adaptability, motivation, format \&layout, usability, accessibility, visibility, and compatibility. Their sample involved over 60 English learning apps which were ranked using the scale from 1 to 5 by two evaluators. They found that interactivity and adaptability were the weakest features. This means that mobile apps need to be improved to cater to learner's interactivity and adaptability needs.

Heil, Wu, Lee and Schmidt (2016) evaluated 55 commercially available mobile language learning apps using a wide range of criteria. The major findings of their study are: the apps teach vocabulary in isolated units instead of presenting them 
in relevant contexts; the apps are minimally suitable for individual learner's set of skills; the apps barely provide explanatory feedback to the users; and the apps mostly lend support to behaviorist learning approach. They recommend more contextualized language, explanatory feedback, and adaptive technology which could make them align with second language acquisition and L2 pedagogical research.

After analyzing five mobile language learning apps, Martín-Monjeand Rodríguez-Arancón (2014) reported that the technical and pedagogic qualities of apps were not consistent with linguistic value and adequacy for language teaching and learning. Sung, Chang and Liu (2016) conducted a meta-analysis and research synthesis study on the previous researches on mobile devices usage in education carried out in recent couple of decades. Their study analyzed 110 articles and found that the impact of software and hardware designed for mobile devices were more effective than that of laptop. This might highlight the importance of exploration of features and effectiveness of mobile apps for language learning.

Kim and Kwon (2012) discussed the features and effectiveness of 87 ESL mobile apps. They investigated the common and distinctive features of mobile apps as well as their strength and weakness for effective mobile assisted mobile language learning (MALL). They found that ESL apps seem to be effective in providing personal and learner-centered opportunities where they are accessible and offer flexible practices. They argue that mobile apps need improvement in terms of being more situated and field dependent and support collaborative learning. They suggest that further studies are needed to be conducted to explore the effectiveness of mobile apps. The result of study shows that mostly apps deal with short language information and most of them need cognitive language learning style. Moreover, L2 approaches are limited and are basically form-focused. Furthermore, most of instructions in the apps of grammar and vocabulary tend to evaluate the linguistic knowledge of individuals than teaching than how to use the language communicatively.

The main objective of Chen's (2016) research study is to assess English-learning mobile apps and their effectiveness in terms of assisting adult learners with limited English proficiency in second language learning. The study involved 232 registered apps which were downloaded and eventually 7 English learning apps were chosen after filtering by reviews and ratings. Data was analyzed through selection and categorization. The study found that mobile apps help adult learners enhance language skills with multiple channels and modalities though one app could not cater to all needs of language learner. He recommends studies on mobile apps for nonnative speakers.

The primary purpose of a study by Deng and Trainin's (2015) is to explore and illustrate theory-based vocabulary learning mobile apps that help make English vocabulary acquisition possible in and out of classroom. The study examined three apps namely, USA TODAY, NPR News and Kindle. The result of the study indicates that there is a high level of usage of mobile devices in educational setting helping students to become a proficient learner. However, new apps usually create uncertainty among students as well as teachers in terms of usage for the purpose of improvement in language learning which could hinder their usage. 
However, the discussion about the effectiveness of mobile applications for language teaching and learning has not been settled yet. Some questions such as the effectiveness of mobile apps from the second language acquisition perspective and the effectiveness of their design for language teaching and learning have not been addressed properly (Kim \& Kwon, 2012). Some researchers have studied and discussed MALL but only as a part of computer assisted language learning. Some features of mobile apps distinguish MALL form CALL (Kim \& Kwon, 2012; Kukulska - Hulme \& Shield, 2008); hence, a study specifically focusing on MALL is needed.

Further, mobile app usage for language learning is unsatisfactory (Kim \& Kwon, 2012). Little knowledge on mobile language learning (MLL) applications is available. The findings of previous studies might not be applicable to the newly developed applications. It is not clear whether the existing mobile language learning apps contribute to MALL or not. Besides, previous studies have mainly focused on IOS system (Kim \& Kwon, 2012) with very few studies, if any, were conducted on apps based on Android.

To address these gaps, the current study has selected ten latest MLL apps for grammar learning based on the rankings provided by online users ( 4.5 and above) and the high number of installations. The purpose of the present study is to deepen the discussion and our understanding of the success of language learning apps in supporting MALL by involving the appropriate features of MALL. To this end, the present study selects 10 currently available mobile apps for learning and improving grammar. The study will focus on the apps supported by Android system.

\section{MALL and Language Learning Theories}

The contribution of second language acquisition and pedagogical research indispensably structure the effective instructional design. It is argued that vast studies have been conducted on different aspects of second language learning; however, when it comes to the development of technology for supporting language learning, the pertinent theories and empirical findings are subject to being overlooked (Kukulska-Hume \& Bull, 2009). A framework proposed by Reinders and Pegrum (2016) for assessing mobile apps reflect the significance of discussion of findings of second language learning and pedagogy when assessing apps. Gaining communicative competence in the second language requires comprehensible input, comprehensible output, negotiating for meaning, and noticing new language promoted through feedback.

Based on theoretical models of language learning, language knowledge covers grammatical knowledge, discourse knowledge, pragmatic knowledge, sociolinguistic knowledge and functional knowledge to name some. A multi range of knowledge is needed to build communicative competence. Hence, the pedagogical approaches to developing apps should answer the questions of what content and how to assess.

So far, several language teaching methods have been introduced such as grammar translation method and audio-lingualism which are characterized as behaviorism in nature since they focus on the skills such as drilling practice memorization and 
repetition (Brown, 2007). Based on the premise of behaviorism, learning takes place as result of association of stimulus-response, which constructs a repository of knowledge in the language learners that could be strengthened or weakened according to the frequency of reinforcement (Fosnot \& Perry, 1996). However, behaviorism approach is missing the social element; this means that it does not consider language use as a fundamentally communicative act. Another important learning theory is cognitivism. It focuses on the learning process instead of on the observed behavior. Based on the tenet of cognitivism, knowledge is regarded as a schema or/and symbolic mental construction and subsequently learning is result of change in learner's schemata (Ormrod \& Davis, 2004).

In sharp contrast with behaviorism, supporters of constructivism reject the idea that human knowledge is directly reflected from an objective reality (Blyth, 2007). Constructivism does not accept the idea that knowledge is transferred from teacher to student. Knowledge is constructed by the process which blends the learner's previous, preexisting knowledge framework with that knowledge encountered in social contexts. An individual Learner learns by being an active member of the surrounding community and the world in general (Oxford, 1995).

Essentially, three dominant theories in instructional environments are behaviorism, cognitivism, and constructivism. However, these theories were introduced prior to the impact of technology and internet on learning. The use of these theories in the context of technology for education and digital learning has limitation in terms of creating, preserving, and using knowledge. This has given rise to the emergence of an internet based learning theory called connectivism introduced by George Siemens and Stephen Downes in 2005. Connectivism is the incorporation of principles of chaos, complexity, network, and self-organization theories (Siemens, 2008). However, there is no clear picture of the application of connectivism in the context of MALL.

With these in mind, the current study attempts to analyze the grammar apps to see how they are embedding the principles of the abovementioned theories, which help learners in acquiring language. It also discusses their usage for practicing MALL. This study characterizes the currently available apps to make recommendations which might be of help for future mobile app development.

\section{Learning Grammar through Mobile Apps}

Grammatical points could be improved or learnt through the programs which are specifically designed and installed on mobile devices or smart phones. Some apps have assessment and testing system where grammatical rules are explained and exercises and quizzes are offered in the form of multiple choice questions, truefalse, fill in the blanks to be responded by the language learners (Miangah \& Nezarat, 2012). Some apps give immediate explanatory feedbacks to the users (Heil, Wu, Lee \& Schmidt, 2016). Given the importance of grammar in the process of language learning, the current study focuses on the assessment of current openly available grammar apps.

\section{Method}


Grammatical points could be improved or learnt through the programs which are specifically designed and installed on mobile devices or smart phones. Some apps have assessment and testing system where grammatical rules are explained and exercises and quizzes are offered in the form of multiple choice questions, truefalse, fill in the blanks to be responded by the language learners (Miangah \& Nezarat, 2012). Some apps give immediate explanatory feedbacks to the users (Heil, Wu, Lee \& Schmidt, 2016). Given the importance of grammar in the process of language learning, the current study focuses on the assessment of current openly available grammar apps.

\section{Theoretical Model}

The study adapts a framework proposed by Supyan Hussin (2013) which takes in to account the learning theories as well as the findings of relevant studies in the era of second language acquisition. The adapted framework has four components namely, System, Program, Curriculum and Language \& Culture, which are illustrated in the following diagram. To triangulate data collocation and analysis, the study also adds the element of reviewers' rating and downloads of the apps based on Google Play Store information.In addition, the study recruited seven experts with background in applied linguistics and IT to evaluate the 10 selected grammar apps using the following framework. Table 1 demonstrates the framework components.

Table 1. The adapted framework

\begin{tabular}{|c|c|c|}
\hline Criteria & Description & $\begin{array}{l}\text { Assessment } \\
\text { Scale }\end{array}$ \\
\hline \multirow[t]{3}{*}{$\begin{array}{l}\text { System (Supyan } \\
\text { Hussin , 2013) }\end{array}$} & - Execution Time: the time needed to run apps & $\begin{array}{l}\text { Normal/not } \\
\text { normal }\end{array}$ \\
\hline & $\begin{array}{l}\text { - Advert\&bugs: advertisements and bugs that } \\
\text { appear while using apps }\end{array}$ & Yes/No \\
\hline & $\begin{array}{l}\text { - Free/paid: refers to the status of being free of } \\
\text { paid apps }\end{array}$ & $\begin{array}{l}\text { Free/Partially } \\
\text { Free/Paid }\end{array}$ \\
\hline \multirow[t]{5}{*}{$\begin{array}{l}\text { Program } \\
\text { (Supyan Hussin } \\
\text { 2013) }\end{array}$} & $\begin{array}{l}\text { key concept presentation: refers to the } \\
\text { presentation of definition and explanation of } \\
\text { app }\end{array}$ & $\begin{array}{l}\text { Zero, Poor, Fair, } \\
\text { Good, Very } \\
\text { Good, Excellent }\end{array}$ \\
\hline & $\begin{array}{l}\text { - Guide: refers to the proper examples } \\
\text { provided. }\end{array}$ & $\begin{array}{l}\text { Zero, Poor, Fair, } \\
\text { Good, Very } \\
\text { Good, Excellent }\end{array}$ \\
\hline & $\begin{array}{l}\text { Drill \& practice: refers to the drills and } \\
\text { practices provided by the app }\end{array}$ & $\begin{array}{l}\text { Zero, Poor, Fair, } \\
\text { Good, Very } \\
\text { Good, Excellent }\end{array}$ \\
\hline & $\begin{array}{l}\text { - Simulation \&problem solving: is associated } \\
\text { with simulating \& problem solving situation }\end{array}$ & $\begin{array}{l}\text { Zero, Poor, Fair, } \\
\text { Good, Very } \\
\text { Good, Excellent }\end{array}$ \\
\hline & $\begin{array}{l}\text { - Testing \& assessment: refers to the } \\
\text { assessment and testing of user's performance }\end{array}$ & $\begin{array}{l}\text { Zero, Poor, Fair, } \\
\text { Good, Very } \\
\text { Good, Excellent }\end{array}$ \\
\hline
\end{tabular}




\begin{tabular}{|c|c|c|}
\hline \multirow[t]{3}{*}{$\begin{array}{l}\text { Curriculum } \\
\text { (Supyan } \\
\text { Hussin, 2013) }\end{array}$} & $\begin{array}{l}\text { - Learning sequence: refers to the sequence of } \\
\text { grammatical points presented. }\end{array}$ & $\begin{array}{l}\text { Zero, Poor, Fair, } \\
\text { Good, Very } \\
\text { Good, Excellent }\end{array}$ \\
\hline & $\begin{array}{l}\text { Skill development: refers to the development } \\
\text { of grammar ability and skill }\end{array}$ & $\begin{array}{l}\text { Zero, Poor, Fair, } \\
\text { Good, Very } \\
\text { Good, Excellent }\end{array}$ \\
\hline & $\begin{array}{l}\text { - MALL Role: refers to the use of app for doing } \\
\text { MALL }\end{array}$ & $\begin{array}{l}\text { Zero, Poor, Fair, } \\
\text { Good, Very } \\
\text { Good, Excellent }\end{array}$ \\
\hline \multirow{5}{*}{$\begin{array}{l}\text { Language \& } \\
\text { Culture } \\
\text { (Supyan } \\
\text { Hussin, 2013) }\end{array}$} & $\begin{array}{l}\text { Autonomous : is associated with the use of } \\
\text { app for self-directed learning }\end{array}$ & $\begin{array}{l}\text { Zero, Poor, Fair, } \\
\text { Good, Very } \\
\text { Good, Excellent }\end{array}$ \\
\hline & $\begin{array}{l}\text { - Audience level: refers to the proficiency level } \\
\text { of user }\end{array}$ & $\begin{array}{l}\text { Basic, } \\
\text { Elementary, } \\
\text { Intermediate, } \\
\text { Advanced }\end{array}$ \\
\hline & $\begin{array}{l}\text { - Complexity level: refers to the difficulty and } \\
\text { order of materials }\end{array}$ & $\begin{array}{l}\text { Zero, Poor, Fair, } \\
\text { Good, Very } \\
\text { Good, Excellent }\end{array}$ \\
\hline & $\begin{array}{l}\text { - Cultural elements: refers to graphics and } \\
\text { symbols connected with culture of target } \\
\text { language }\end{array}$ & $\begin{array}{l}\text { Zero, Poor, Fair, } \\
\text { Good, Very } \\
\text { Good, Excellent }\end{array}$ \\
\hline & $\begin{array}{l}\text { Spelling \& grammar accuracy: refers to the } \\
\text { accuracy of content in terms of spelling and } \\
\text { grammar }\end{array}$ & $\begin{array}{l}\text { Zero, Poor, Fair, } \\
\text { Good, Very } \\
\text { Good, Excellent }\end{array}$ \\
\hline \multirow{8}{*}{$\begin{array}{l}\text { App users' } \\
\text { Reviews, } \\
\text { Ratings and } \\
\text { Downloads } \\
\text { (https://suppo } \\
\text { rt.google.com/ } \\
\text { googleplay/) }\end{array}$} & $\begin{array}{l}\text { Reviewers: In Google Play Console, individual } \\
\text { users provide reviews about an app. They can } \\
\text { rate with a star rating and review. However, they } \\
\text { can only rate an app once though they can update } \\
\text { their reviews and rating at any time. }\end{array}$ & $\begin{array}{l}\text { Number of } \\
\text { reviewers }\end{array}$ \\
\hline & $\begin{array}{l}\text { Star rating: In Google Play store, users can rate an } \\
\text { app from one to five stars. It is regarded as a } \\
\text { simple average percentage of an app's quality } \\
\text { given by the app users. The counting of stars are } \\
\text { divided in the following categories: }\end{array}$ & \multirow{6}{*}{$\begin{array}{l}\text { From one to } \\
\text { five }\end{array}$} \\
\hline & 5 Stars $=$ Excellent & \\
\hline & 4 Stars=Good & \\
\hline & 3 Stars $=\mathrm{Ok}$ & \\
\hline & 2 Stars $=$ Bad & \\
\hline & 1 Star $=$ Worst & \\
\hline & $\begin{array}{l}\text { Content or age Rated:Ratings are typically } \\
\text { according to a number of factors, such as sexual } \\
\text { content, drugs, violence, gambling, as well as } \\
\text { profane language. }\end{array}$ & $\begin{array}{l}\text { Based on } \\
\text { Google Rating } \\
\text { standards by } \\
\text { country or } \\
\text { region: }(3+, 7+\text {, } \\
12+.16+, \& 18+)\end{array}$ \\
\hline
\end{tabular}


Downloads: The number of People who downloaded and installed the app on their smart phone.

The number of downloads

\section{Findings}

This section presents the findings of user ratings of ten (10) mobile apps that are openly available for learning and improving English grammar based on the adapted framework, which are discussed subsequently. It is shown that "Learn English Grammar" and "English Grammar Book" apps scored highest (4.7/5 star rating) among all ten(10) analyzed apps, while "Oxford Learner's Quick Grammar" scored the lowest which is 3.7/5 star rating. Table 2 presents the analysis of mobile apps in terms of system and program.

Table 2. Reviewers Ratings and Downloads

\begin{tabular}{lcccc}
\hline Grammar Application & Reviewers & $\begin{array}{c}\text { Star } \\
\text { rating }\end{array}$ & $\begin{array}{c}\text { Content or } \\
\text { age Rated }\end{array}$ & Downloads \\
\hline English & 137 & 4.2 & $3+$ & $100 \mathrm{~K}+$ \\
Grammar & & & & \\
Book & & & & \\
\hline
\end{tabular}

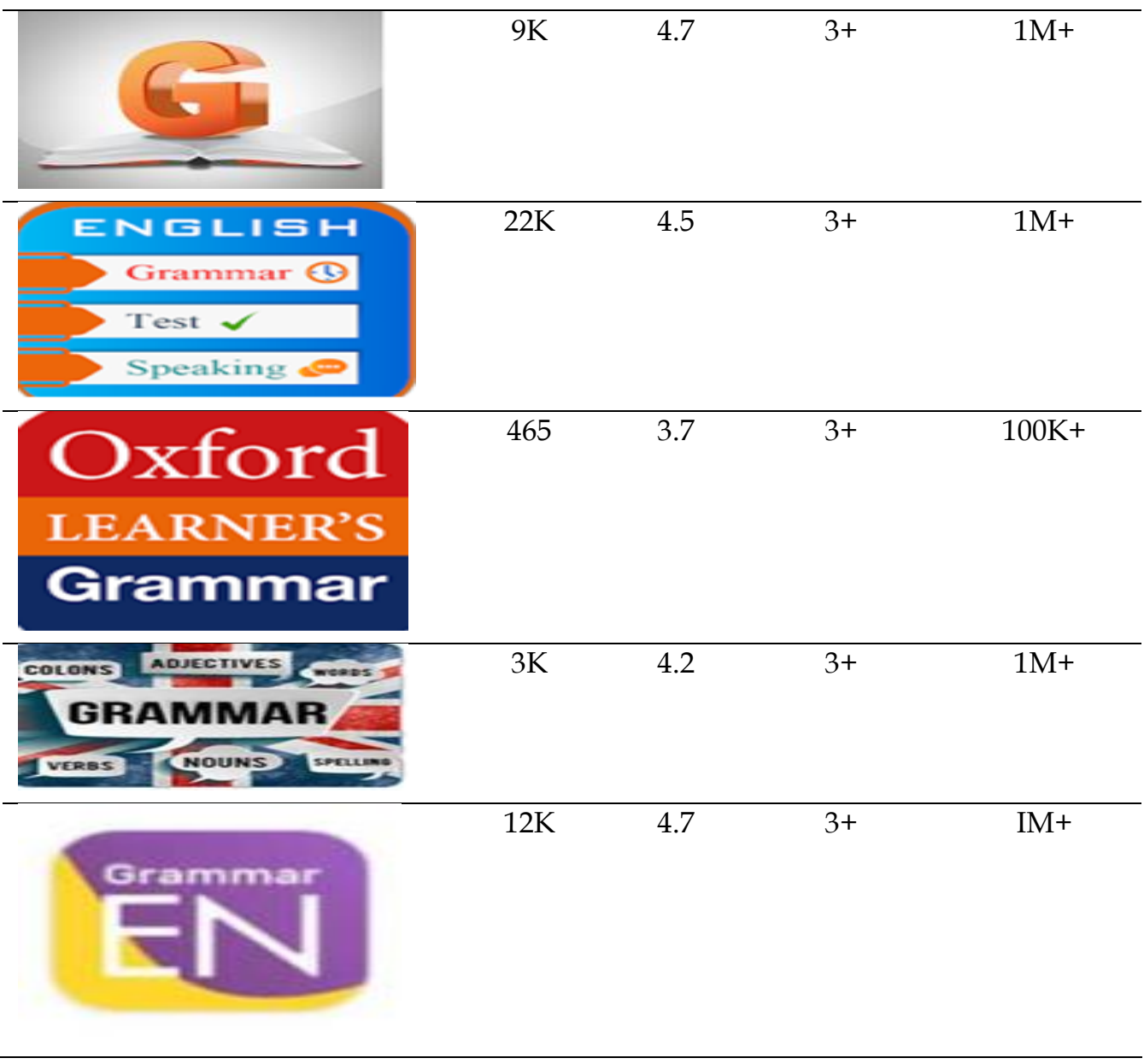




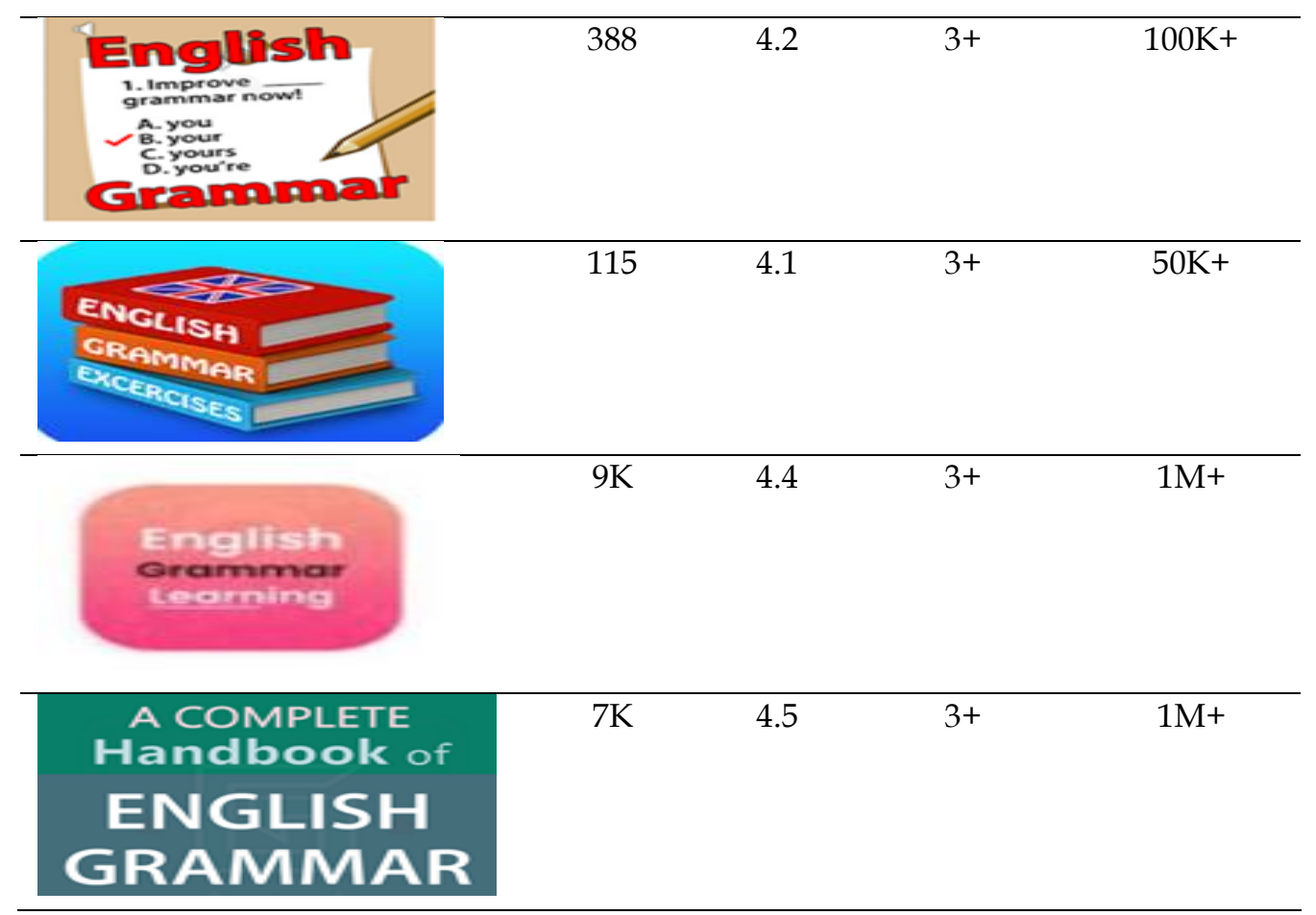

As illustrated in Table 2, the reviewers' views concerning app star are provided. The number of apps downloads are also demonstrated. All apps were rated 3+ which means that they are suitable for all age groups; however, some violence in a comical or fantasy context could be acceptable but bad language is not permitted. In terms of reviews and ratings, App 2 with $9 \mathrm{~K}$ and App 6 with $12 \mathrm{~K}$ reviewers have been given the highest number of stars (4.7), while App 4 with 465 reviewers has been given the lowest number of stars (3.7). App 8 with 115 reviewers was rated as 4.1 star app and App 7 with 388 reviewers was rated as 4.2 stars, App 1 with 137 reviewers was rated as 4.2. APP 3 with $22 \mathrm{~K}$ viewers was rated as 4.5 star app. App 9 and App 10 with $9 \mathrm{~K}$ and $7 \mathrm{~K}$ reviewers have ratings of 4.4 and 4.5 stars respectively (as of June 2021). However, while rated based on star number might reveal some facts about users' satisfaction; some apps had very few numbers of reviewers compared to others which may undermine the validity of stars given. In terms of downloads, Apps 2, 3, 5, 6, 9 and 10 have been downloaded by more than million people. Apps 1 and 4 have been downloaded by $100 \mathrm{~K}+$ users, while App 8 has only been downloaded by $50 \mathrm{~K}+$ people. The apps with higher number of downloads have greater number of reviewers and as a result their rating maybe more reliable.

Table 3. Features Analysis on Apps (System and Program)

\begin{tabular}{|c|c|c|c|c|c|c|c|c|}
\hline \multirow[t]{2}{*}{ App } & \multicolumn{3}{|c|}{ System } & \multicolumn{5}{|c|}{ Program } \\
\hline & 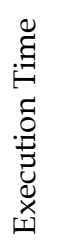 & 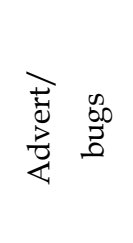 & 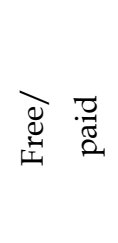 & 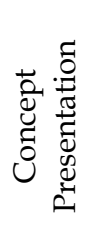 & $\begin{array}{l}\frac{0}{0} \\
: 0 \\
0\end{array}$ & 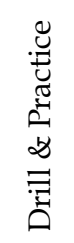 & 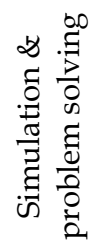 & 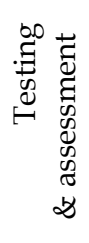 \\
\hline
\end{tabular}




\begin{tabular}{|c|c|c|c|c|c|c|c|c|}
\hline $\begin{array}{l}\text { English } \\
\text { Grammar } \\
\text { Book }\end{array}$ & $\begin{array}{l}\text { Normal } \\
100 \% \\
\text { Not } \\
\text { normal } \\
(0 \%)\end{array}$ & $\begin{array}{l}\text { Yes } \\
100 \% \\
\text { No } \\
0 \%\end{array}$ & $\begin{array}{l}\text { Free } \\
100 \% \\
\text { partially } \\
\text { free } 0 \% \\
\text { paid } 0 \%\end{array}$ & $\begin{array}{l}\text { Zero } \\
0 \%, \\
\text { Poor } \\
0 \%, \text { fair } \\
14 \% \text {, } \\
\text { Good, } \\
72 \%, \\
\text { very } \\
\text { good } \\
14 \%, \\
\text { excellen } \\
\text { t } 0 \%\end{array}$ & $\begin{array}{l}\text { Zero } \\
0 \%, \\
\text { Poor } \\
0 \%, \text { fair } \\
14 \%, \\
\text { Good } \\
72 \%, \\
\text { very } \\
\text { good } \\
14 \%, \\
\text { excellen } \\
\text { t } 0 \%\end{array}$ & $\begin{array}{l}\text { Zero } \\
0 \%, \\
\text { Poor } \\
0 \%, \text { fair } \\
14 \%, \\
\text { Good72 } \\
\%, \text { very } \\
\text { xgood1 } \\
4 \%, \\
\text { excellen } \\
\text { t0\% }\end{array}$ & $\begin{array}{l}\text { Zero } \\
100 \%, \\
\text { Poor } \\
0 \%, \\
\text { Fair 0\%, } \\
\text { Good } \\
0 \%, \\
\text { very } \\
\text { good } \\
0 \%, \\
\text { excellen } \\
\text { t } 0 \%\end{array}$ & $\begin{array}{l}\text { Zero100 } \\
\%, \text { Poor } \\
0 \% \\
\text {, fair } \\
0 \%, \\
\text { Good } \\
0 \% \text {, } \\
\text { very } \\
\text { good } \\
0 \%, \\
\text { excellen } \\
\text { t } 0 \%\end{array}$ \\
\hline (2) & $\begin{array}{l}\text { Normal } \\
100 \% \\
\text { Not } \\
\text { normal } \\
0 \%\end{array}$ & $\begin{array}{l}\text { Yes } \\
100 \% \\
\text { No } \\
0 \%\end{array}$ & $\begin{array}{l}\text { Free100\% } \\
\text { Partially } \\
\text { Free 0\% } \\
\text { Paid 0\% }\end{array}$ & $\begin{array}{l}\text { Zero0\% } \\
\text {, } \\
\text { Poor0\% } \\
\text {, Fair } \\
14 \%, \\
\text { Good72 } \\
\%, \text { Very } \\
\text { Good } \\
14 \%, \\
\text { Excelle } \\
\text { nt } 0 \%\end{array}$ & $\begin{array}{l}\text { Zero0\% } \\
\text { ' } \\
\text { Poor0\% } \\
\text { Fair28\% } \\
\text { Good72 } \\
\%, \text { Very } \\
\text { Good0 } \\
\%, \\
\text { Excelle } \\
\text { nt0\% }\end{array}$ & $\begin{array}{l}\text { Zero0\% } \\
\text { ' } \\
\text { Poor0\% } \\
\text { Fair14\% } \\
\text { ' Good72 } \\
\%, \text { Very } \\
\text { Good14 } \\
\%, \\
\text { Excelle } \\
\text { nt0\% }\end{array}$ & $\begin{array}{l}\text { Zero0\% } \\
\text { Poor0\% } \\
\text { ' } \\
\text { Fair28\% } \\
\text { ' Good72 } \\
\%, \\
\text { Very } \\
\text { good0\% } \\
\text { Excelle } \\
\text { nt0\% }\end{array}$ & $\begin{array}{l}\text { Zero0\% } \\
\text { Poor0\% } \\
\text { ' Fair14\% } \\
\text { ' Good86 } \\
\%, \text { Very } \\
\text { Good } \\
0 \%, \\
\text { Excelle } \\
\text { nt 0\% }\end{array}$ \\
\hline (3) & $\begin{array}{l}\text { Not } \\
\text { normal } \\
0 \%\end{array}$ & $\begin{array}{l}\text { Yes10 } \\
0 \%, \\
\text { No } \\
0 \%\end{array}$ & $\begin{array}{l}\text { Free100\% } \\
\text { Partially } \\
\text { Free0\%, } \\
\text { Paid0\% }\end{array}$ & $\begin{array}{l}\text { Zero0\% } \\
\text { ' } \\
\text { Poor0\% } \\
\text { ' Fair28\% } \\
\text { ' Good72 } \\
\text { \%, Very } \\
\text { Good0 } \\
\%, \\
\text { Excelle } \\
\text { nt0\% }\end{array}$ & $\begin{array}{l}\text { Zero0\% } \\
\text { Poor0\% } \\
\text { ' } \\
\text { Fair14\% } \\
\text { Good72 } \\
\%, \text { Very } \\
\text { Good14 } \\
\%, \\
\text { Excelle } \\
\text { nt0\% }\end{array}$ & $\begin{array}{l}\text { Zero0\% } \\
\text { ' } \\
\text { Poor0\% } \\
\text { Fair14\% } \\
\text { ' Good86 } \\
\%, \text { Very } \\
\text { Good0 } \\
\%, \\
\text { Excelle } \\
\text { nt0\% }\end{array}$ & $\begin{array}{l}\text { Zero0\% } \\
\text { ' } \\
\text { Poor0\% } \\
\text { Fair14\% } \\
\text { ' } \\
\text { Good72 } \\
\%, \\
\text { Very } \\
\text { Good14 } \\
\%, \\
\text { Excelle } \\
\text { nt0\% }\end{array}$ & $\begin{array}{l}\text { Zero0\% } \\
\text { Poor0\% } \\
\text { ' Fair14\% } \\
\text { Good72 } \\
\%, \text { Very } \\
\text { Good14 } \\
\%, \\
\text { Excelle } \\
\text { nt0\% }\end{array}$ \\
\hline LEARN & $\begin{array}{l}\text { Normal } \\
0 \% \\
\text { Not } \\
\text { normal1 } \\
00 \%\end{array}$ & $\begin{array}{l}\text { Yes10 } \\
0 \% \\
\text { No } \\
0 \%\end{array}$ & $\begin{array}{l}\text { Free0\%, } \\
\text { Partially } \\
\text { Free100\% } \\
\text { Paid0\% }\end{array}$ & $\begin{array}{l}\text { Zero0\% } \\
\text { ' } \\
\text { Poor0\% } \\
\text { Fair14\% } \\
\text { ', Good72 } \\
\%, \text { Very } \\
\text { Good14 } \\
\%, \\
\text { Excelle } \\
\text { nt0\% }\end{array}$ & $\begin{array}{l}\text { Zero0\% } \\
\text { ' } \\
\text { ' } \\
\text { Fair14\% } \% \\
\text { ' Good86 } \\
\%, \text { Very } \\
\text { Good0 } \\
\%, \\
\text { Excelle } \\
\text { nt0\% }\end{array}$ & $\begin{array}{l}\text { Zero0\% } \\
\text { ' } \\
\text { Poor0\% } \\
\text { Fair14\% } \\
\text { ', Good72 } \\
\%, \text { Very } \\
\text { Good14 } \\
\%, \\
\text { Excelle } \\
\text { nt0\% }\end{array}$ & $\begin{array}{l}\text { Zero0\% } \\
\text { ' } \\
\text { Poor0\% } \\
\text { Fair86\% } \\
\text { ', } \\
\text { Good14 } \\
\%, \\
\text { Very } \\
\text { Good0 } \\
\%, \\
\text { Excelle } \\
\text { nt0\% }\end{array}$ & $\begin{array}{l}\text { Zero100 } \\
\%, \\
\text { Poor0\% } \\
\text { ' } \\
\text { Fair0\%, } \\
\text { Good0 } \\
\%, \text { Very } \\
\text { Good0 } \\
\%, \\
\text { Excelle } \\
\text { nt0\% }\end{array}$ \\
\hline
\end{tabular}




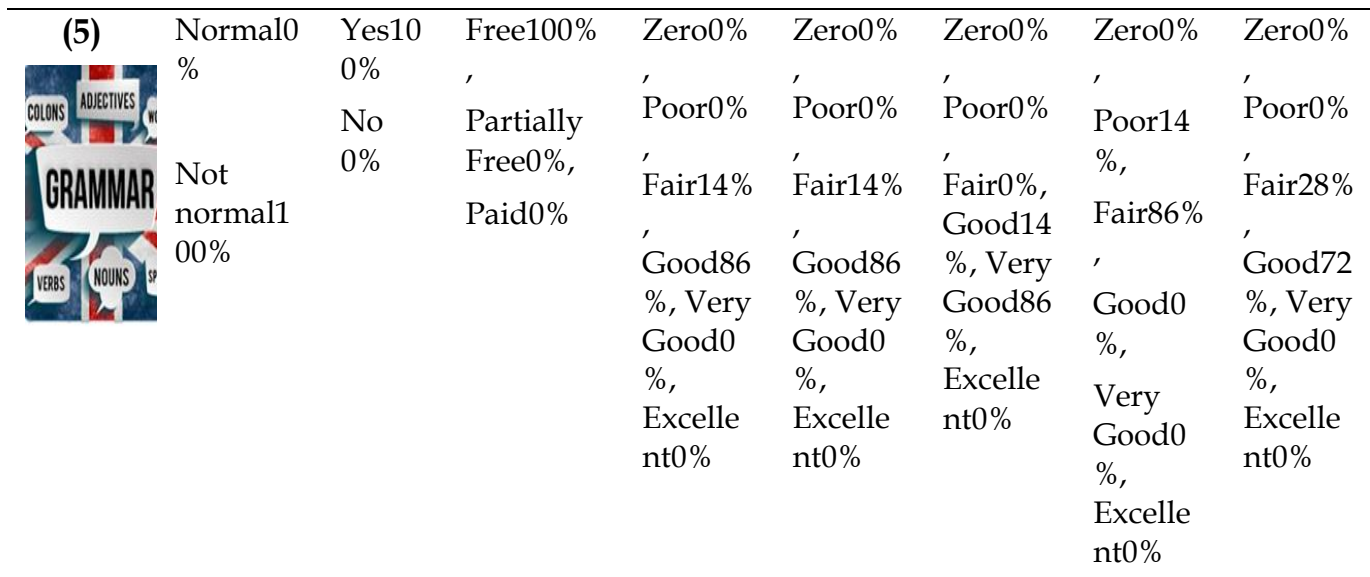

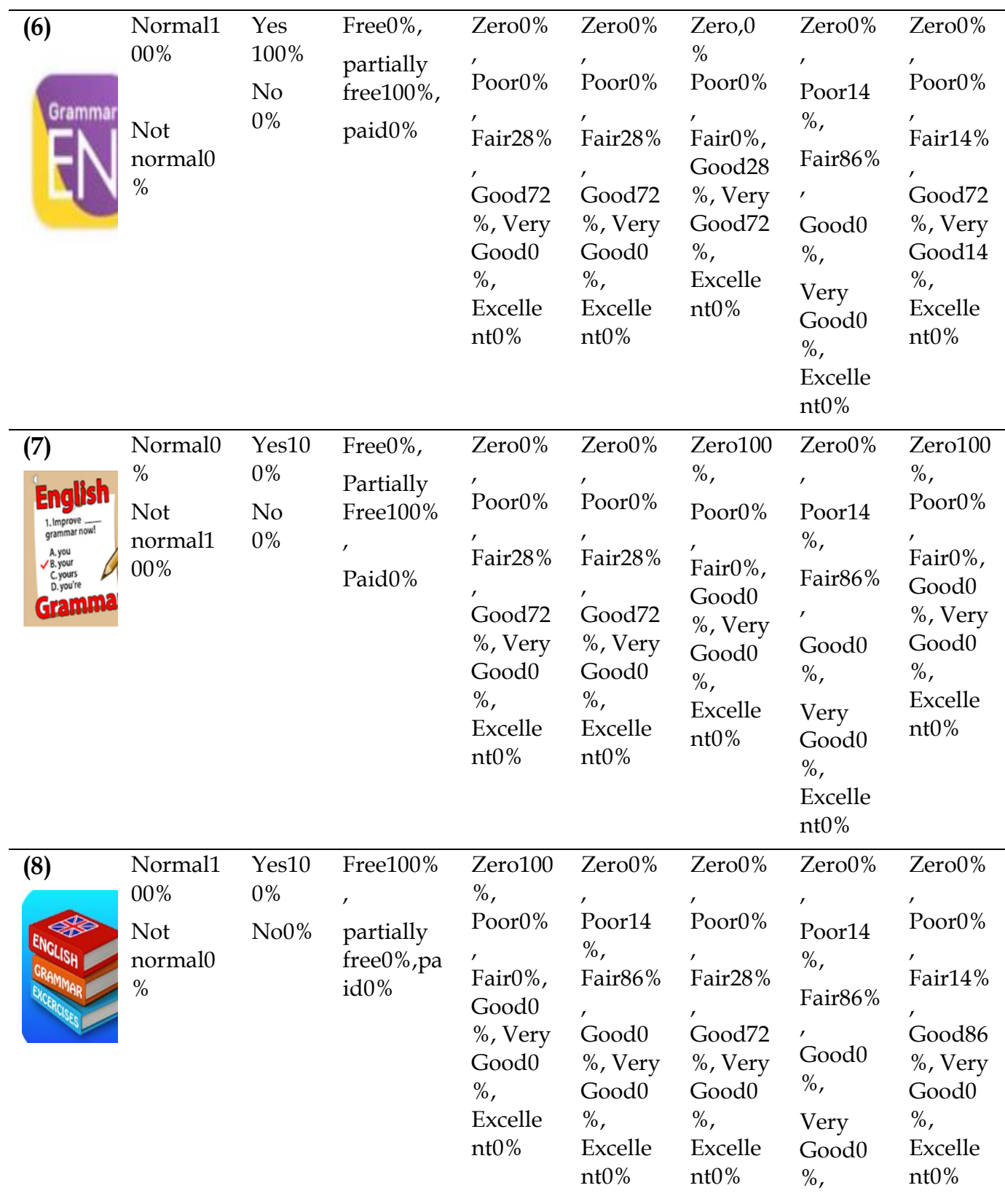




\begin{tabular}{|c|c|c|c|c|c|c|c|c|}
\hline & & & & & & & $\begin{array}{l}\text { Excelle } \\
\text { nt } 0 \%\end{array}$ & \\
\hline $\begin{array}{l}\text { English } \\
\text { Grammar } \\
\text { Coorning }\end{array}$ & $\begin{array}{l}\text { Normal1 } \\
00 \% \\
\text { Not } \\
\text { normal0 } \\
\%\end{array}$ & $\begin{array}{l}\text { Yes } \\
100 \% \\
\text { No } \\
0 \%\end{array}$ & $\begin{array}{l}\text { Free0\% } \\
\text { Partially } \\
\text { Free100\% } \\
\text { Paid0\% }\end{array}$ & $\begin{array}{l}\text { Zero0\% } \\
\text { Poor0\% } \\
\text { ' Fair28\% } \\
\text { ' Good72 } \\
\%, \text { Very } \\
\text { Good0 } \\
\%, \\
\text { Excelle } \\
\text { nt0\% }\end{array}$ & $\begin{array}{l}\text { Zero0\% } \\
\text { Poor0\% } \\
\text { 'Fair14\% } \\
\text { ' Good86 } \\
\%, \text { Very } \\
\text { Good0 } \\
\%, \\
\text { Excelle } \\
\text { nt0\% }\end{array}$ & $\begin{array}{l}\text { Zero0\% } \\
\text { ' } \\
\text { ' } \\
\text { Fair14\% } \% \\
\text { ' Good72 } \\
\%, \text { Very } \\
\text { Good14 } \\
\%, \\
\text { Excelle } \\
\text { nt0\% }\end{array}$ & $\begin{array}{l}\text { Zero0\% } \\
\text {, } \\
\text { Poor0\% } \\
\text { ' } \\
\text { Fair14\% } \\
\text { ', } \\
\text { Good86 } \\
\%, \\
\text { Very } \\
\text { Good0 } \\
\%, \\
\text { Excelle } \\
\text { nt0\% }\end{array}$ & $\begin{array}{l}\text { Zero0\% } \\
\text { Poor0\% } \\
\text { Fair14\% } \\
\text { ' } \\
\text { Good } \\
86 \%, \\
\text { Very } \\
\text { Good0 } \\
\%, \\
\text { Excelle } \\
\text { nt0\% }\end{array}$ \\
\hline $\begin{array}{l}\text { (10) } \\
\text { A COMPLETE } \\
\text { Handbook } \\
\text { ENGIISF } \\
\text { GRAMMA }\end{array}$ & $\begin{array}{l}\text { Normal1 } \\
00 \% \\
\text { Not } \\
\text { normal0 } \\
\%\end{array}$ & $\begin{array}{l}\text { Yes10 } \\
0 \% \\
\text { No0\% }\end{array}$ & $\begin{array}{l}\text { Free100\% } \\
\text { Partially } \\
\text { Free0\%, } \\
\text { Paid0\% }\end{array}$ & $\begin{array}{l}\text { Zero0\% } \\
\text { ' Poor0\% } \\
\text { ' Fair14\% } \\
\text { ' Good72 } \\
\%, \text { Very } \\
\text { Good14 } \\
\%, \\
\text { Excelle } \\
\text { nt0\% }\end{array}$ & $\begin{array}{l}\text { Zero0\% } \\
\text { ' Poor0\% } \\
\text { ' Fair14\% } \\
\text { ' Good86 } \\
\%, \text { Very } \\
\text { Good0 } \\
\%, \\
\text { Excelle } \\
\text { nt0\% }\end{array}$ & $\begin{array}{l}\text { Zero100 } \\
\%, \\
\text { Poor0\% } \\
\text { ' } \\
\text { Fair0\%, } \\
\text { Good0 } \\
\%, \text { Very } \\
\text { Good0 } \\
\%, \\
\text { Excelle } \\
\text { nt0\% }\end{array}$ & $\begin{array}{l}\text { Zero0\% } \\
\text {, } \\
\text { Poor14 } \\
\%, \\
\text { Fair86\% } \\
\text { ' } \\
\text { Good0 } \\
\%, \\
\text { Very } \\
\text { Good0 } \\
\%, \\
\text { Excelle } \\
\text { nt0\% }\end{array}$ & $\begin{array}{l}\text { Zero100 } \\
\%, \\
\text { Poor0\% } \\
\text { Fair0\%, } \\
\text { Good0 } \\
\%, \\
\text { Very } \\
\text { Good0 } \\
\%, \\
\text { Excelle } \\
\text { nt0\% }\end{array}$ \\
\hline
\end{tabular}

As demonstrated in Table 3, the system and programs of the apps were analyzed. Regarding the system, the apps are mostly free except for apps 4, 6, 7, and 9 which are partially free. It is seen that most of the apps have normal execution time for running, except for apps 4,5 and 7. It is noted that all apps have advertisements which sometimes could be irritating or slowing down the use of app. Concerning the concept presentation in the program, App 1 (fair 14\%, Good 72\%, very good 14\%), app2 (Fair 14\%, Good 72\%, Very Good 14\%), app3 (Fair 28\%, Good 72\%), app4 (Fair 14\%, Good 72\%, Very Good 14\%), app5 (Fair 14\%, Good 86\%), app6 (Fair 28\%, Good 72\%), app7 (Fair 28\%, Good 72\%), app8 (Zero 100\%), app9 (Fair $28 \%$, Good $72 \%$ ), and app10 (Fair 14\%, Good 72\%, Very Good 14\%), overall have good concept presentation as the option 'good' accounts for the big percentage of selections; however, app 8 has no concept presentation.

Regarding app guide, app1 (fair14\%, Good 72\%, very good $14 \%$ ), app2 (Fair 28\%, Good 72\%), app3 (Fair 14\%, Good 72\%, Very Good 14\%), app4 (Fair 14\%, Good 86\%), app5 (Fair 14\%, Good 86\%), app6 (Fair 28\%, Good 72\%), app7 (Fair 28\%, Good 72\%), app8 (Poor 14\%, Fair 86\%), app9 (Fair 14\%, Good 86\%), app10 (Fair $14 \%$, Good $86 \%$ ) most of the experts have selected the highest the option 'good' with app 8 with the lowest guide (fair). It was found that 9 apps have quite good guide and exemplification, while app 8 has a fair guide. 
Another feature is drill and practice in which the evaluators have rated app1 (fair $14 \%$, Good 72\%, very good 14\%), app2 (Fair14\%, Good72\%, Very Good 14\%), app3(Fair 14\%, Good 86\%), app4 (Fair 14\%, Good 72\%, Very Good 14\%), app5 (Good 14\%, Very Good 86\%), app6 (Good 28\%, Very Good 72\%), app7 (Zero 100\%), app8 (Fair 28\%, Good 72\%), app9 (Fair 14\%, Good 72\%, Very Good 14\%), and app10 (Zero 100\%). It is indicated that 8 apps have quite good drill and practice while two apps (7 and 10) have no drill and practice.

Another assessed feature is simulation and problem solving. Evaluators have rated app 1 (Zero 100\%), app 2 (Fair 28\%, Good 72\%), app3 (Fair 14\%, Good 72\%, Very Good 14\%), app4 (Fair 86\%, Good 14\%), app5 (Poor 14\%, Fair 86\%), app6 (Poor 14\%, Fair 86\%), app7 (Poor 14\%, Fair 86\%), app8 (Poor 14\%, Fair 86\%), app9 (Fair 14\%, Good 86\%), app10 (Poor 14\%, Fair 86\%). It was found that 5 apps (4, 5, $6,7 \& 10)$ have are fair in terms of simulation and problem solving, while 4 apps $(2,3,4 \& 9)$ have quite good simulation and problem solving feature. However, app 1 has no simulation and problem solving.

Another feature is testing and assessment. Evaluators have rated app 1(Zero100\%), app2 (Fair14\%, Good86\%), app3 (Fair14\%, Good72\%, Very Good14\%), app4 (Zero 100\%), app5 (Fair 28\%, Good 72\%), app6 (Fair 14\%, Good 72\%, Very Good 14\%), app7 (Zero 100\%), app8 (Fair 14\%, Good 86\%), app9 (Fair $14 \%$, Good 86\%), and app10 (Zero 100\%). The findings show that 4 apps $(1,4,7$, \&10) have no testing and assessment, while 6 apps $(2,3,5,6,8 \& 9)$ have quite good testing and assessment. The following table analyzes the mobile apps in terms of curriculum and language \& culture.

Table 4: Features Analysis on apps (Curriculum and Language \& Culture)

\begin{tabular}{|c|c|c|c|c|c|c|c|c|}
\hline \multirow[t]{2}{*}{ Apps } & \multicolumn{4}{|c|}{ Curriculum } & \multicolumn{4}{|c|}{ Language \& Culture } \\
\hline & 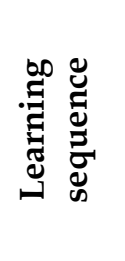 & 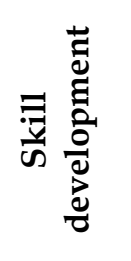 & $\begin{array}{l}\frac{0}{0} \\
\frac{1}{3} \\
\frac{3}{\Sigma}\end{array}$ & 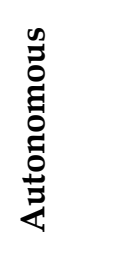 & 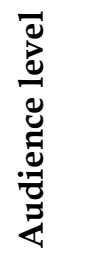 & 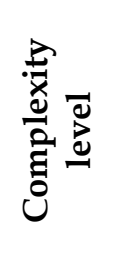 & 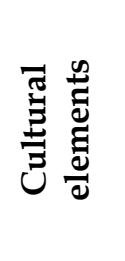 & 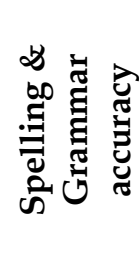 \\
\hline \multirow[t]{2}{*}{ (1) } & Zero & Zero0 & Zero0 & Zero0 & \multirow{11}{*}{$\begin{array}{l}\text { All } \\
\text { levels }\end{array}$} & Zero0 & Zero10 & Zero0 \\
\hline & $0 \%$ & & $\%$ & $\%$ & & $\%$ & $0 \%$ & $\%$ \\
\hline \multirow{9}{*}{$\begin{array}{l}\text { Englis } \\
\text { Gramm } \\
\text { Book }\end{array}$} & \multirow{6}{*}{$\begin{array}{l}\text { Poor } \\
14 \%, \\
\text { Fair } \\
86 \%, \\
\text { Good } \\
0 \%\end{array}$} & Poor0 & Poor0 & \multirow{2}{*}{ Poor0 } & & \multirow{3}{*}{$\begin{array}{l}\text { Poor0 } \\
\%, \\
\text { Fair14 }\end{array}$} & \multirow{3}{*}{$\begin{array}{l}\text { Poor0 } \\
\%, \\
\text { Fair0\% }\end{array}$} & \multirow{4}{*}{$\begin{array}{l}\text { Poor0 } \\
\%, \\
\text { Fair0\% }\end{array}$} \\
\hline & & & $\%$ & & & & & \\
\hline & & Fair14 & Fair14 & \multirow{2}{*}{$\begin{array}{l}\text { Fair14 } \\
\%\end{array}$} & & & & \\
\hline & & $\%$ & $\%$, & & & $\begin{array}{l}\text { Fair14 } \\
\%,\end{array}$ & , & \\
\hline & & Good8 & Good8 & Good8 & & Good7 & Good0 & ' \\
\hline & & $\begin{array}{l}6 \%, \\
\text { Very }\end{array}$ & $\begin{array}{l}6 \%, \\
\text { Verv }\end{array}$ & $\begin{array}{l}\text { 6\%, } \\
\text { Verv }\end{array}$ & & $\begin{array}{l}2 \%, \\
\text { Very }\end{array}$ & $\begin{array}{l}\%, \\
\text { Verv }\end{array}$ & $\begin{array}{l}\text { Good0 } \\
\%\end{array}$ \\
\hline & \multirow{3}{*}{$\begin{array}{l}\text { Good } \\
0 \%, \\
\text { Excelle } \\
\text { nt } 0 \%\end{array}$} & Good0 & Good0 & Good0 & & Good1 & Good0 & Verv \\
\hline & & & $\%$ & $\%$ & & $4 \%$ & $\%$ & Good1 \\
\hline & & $\begin{array}{l}\text { Excelle } \\
\text { nt0\% }\end{array}$ & $\begin{array}{l}\text { Excelle } \\
\text { nt0\% }\end{array}$ & $\begin{array}{l}\text { Excelle } \\
\text { nt0\% }\end{array}$ & & $\begin{array}{l}\text { Excelle } \\
\text { nt } 0 \%\end{array}$ & $\begin{array}{l}\text { Excelle } \\
\text { nt0\% }\end{array}$ & $\begin{array}{l}00 \%, \\
\text { Excelle } \\
\text { nt0\% }\end{array}$ \\
\hline \multirow[t]{4}{*}{ (2) } & Zero & Zero0 & Zero0 & Zero0 & \multirow{4}{*}{$\begin{array}{l}\text { All } \\
\text { levels }\end{array}$} & Zero0 & Zero0 & \multirow{4}{*}{$\begin{array}{l}\text { Zero0 } \\
\%,\end{array}$} \\
\hline & $0 \%$ & & & $\%$, & & $\%$ & $\%$, & \\
\hline & & Poor0 & Poor0 & Poor0 & & Poor0 & Poor0 & \\
\hline & & $\%$, & $\%$, & $\%$, & & $\%$, & $\%$ & \\
\hline
\end{tabular}




\begin{tabular}{|c|c|c|c|c|c|c|c|c|}
\hline 2 & $\begin{array}{l}\text { Poor } \\
0 \%, \\
\text { Fair } \\
14 \%, \\
\text { Good } \\
86 \%, \\
\text { Very } \\
\text { Good } \\
0 \%, \\
\text { Excelle } \\
\text { nt } 0 \%\end{array}$ & $\begin{array}{l}\text { Fair28 } \\
\%, \\
\text { Good7 } \\
2 \%, \\
\text { Very } \\
\text { Good0 } \\
\%, \\
\text { Excelle } \\
\text { nt0\% }\end{array}$ & $\begin{array}{l}\text { Fair14 } \\
\%, \\
\text { Good8 } \\
6 \%, \\
\text { Very } \\
\text { Good0 } \\
\%, \\
\text { Excelle } \\
\text { nt0\% }\end{array}$ & $\begin{array}{l}\text { Fair14 } \\
\%, \\
\text { Good7 } \\
2 \%, \\
\text { Very } \\
\text { Good1 } \\
4 \%, \\
\text { Excelle } \\
\text { nt0\% }\end{array}$ & & $\begin{array}{l}\text { Fair28 } \\
\%, \\
\text { Good7 } \\
2 \%, \\
\text { Very } \\
\text { Good0 } \\
\%, \\
\text { Excelle } \\
\text { nt0\% }\end{array}$ & $\begin{array}{l}\text { Fair28 } \\
\%, \\
\text { Good7 } \\
2 \%, \\
\text { Very } \\
\text { Good0 } \\
\%, \\
\text { Excelle } \\
\text { nt0\% }\end{array}$ & $\begin{array}{l}\begin{array}{l}\text { Poor0 } \\
\%,\end{array} \\
\text { Fair0\% } \\
\text { Good1 } \\
\text { 00\%, } \\
\text { Very } \\
\text { Good0 } \\
\%, \\
\text { Excelle } \\
\text { nt0\% }\end{array}$ \\
\hline $\begin{array}{l}\text { (3) } \\
\text { ENGLISH } \\
\text { Grammar Q } \\
\text { Test } \checkmark \\
\text { Speaking } \\
\end{array}$ & $\begin{array}{l}\text { Zero } \\
0 \%, \\
\text { Poor } \\
0 \%, \\
\text { Fair } \\
0 \%, \\
\text { Good } \\
28 \%, \\
\text { Very } \\
\text { Good } \\
72 \%, \\
\text { Excelle } \\
\text { nt } 0 \%\end{array}$ & $\begin{array}{l}\text { Zero0 } \\
\%, \\
\text { Poor0 } \\
\%, \\
\text { Fair14 } \\
\%, \\
\text { Good8 } \\
6 \%, \\
\text { Very } \\
\text { Good0 } \\
\%, \\
\text { Excelle } \\
\text { nt0\% }\end{array}$ & $\begin{array}{l}\text { Zero0 } \\
\%, \\
\text { Poor0 } \\
\%, \\
\text { Fair0\% } \\
\text { ' } \\
\text { Good1 } \\
00 \%, \\
\text { Very } \\
\text { Good0 } \\
\%, \\
\text { Excelle } \\
\text { nt0\% }\end{array}$ & $\begin{array}{l}\text { Zero0 } \\
\%, \\
\text { Poor0 } \\
\%, \\
\text { Fair14 } \\
\%, \\
\text { Good7 } \\
2 \%, \\
\text { Very } \\
\text { Good1 } \\
4 \%, \\
\text { Excelle } \\
\text { nt0\% }\end{array}$ & $\begin{array}{l}\text { All } \\
\text { levels }\end{array}$ & $\begin{array}{l}\text { Zero0 } \\
\%, \\
\text { Poor0 } \\
\%, \\
\text { Fair0\% } \\
\text { ' } \\
\text { Good2 } \\
8 \%, \\
\text { Very } \\
\text { Good7 } \\
2 \%, \\
\text { Excelle } \\
\text { nt0\% }\end{array}$ & $\begin{array}{l}\text { Zero0 } \\
\%, \\
\text { Poor28 } \\
\%, \\
\text { Fair72 } \\
\%, \\
\text { Good0 } \\
\%, \\
\text { Very } \\
\text { Good0 } \\
\%, \\
\text { Excelle } \\
\text { nt0\% }\end{array}$ & $\begin{array}{l}\text { Zero0 } \\
\%, \\
\text { Poor0 } \\
\%, \\
\text { Fair0\% } \\
\text { Good1 } \\
00 \%, \\
\text { Very } \\
\text { Good0 } \\
\%, \\
\text { Excelle } \\
\text { nt0\% }\end{array}$ \\
\hline $\begin{array}{l}\text { LEARNER'S } \\
\text { Crammar }\end{array}$ & $\begin{array}{l}\text { Zero } \\
0 \%, \\
\text { Poor } \\
0 \%, \\
\text { Fair } \\
14 \%, \\
\text { Good } \\
72 \% \text {, } \\
\text { Very } \\
\text { Good } \\
14 \%, \\
\text { Excelle } \\
\text { nt } 0 \%\end{array}$ & $\begin{array}{l}\text { Zero0 } \\
\%, \\
\text { Poor0 } \\
\%, \\
\text { Fair14 } \\
\%, \\
\text { Good8 } \\
6 \%, \\
\text { Very } \\
\text { Good0 } \\
\%, \\
\text { Excelle } \\
\text { nt0\% }\end{array}$ & $\begin{array}{l}\text { Zero0 } \\
\%, \\
\text { Poor14 } \\
\%, \\
\text { Fair72 } \\
\%, \\
\text { Good1 } \\
4 \%, \\
\text { Very } \\
\text { Good0 } \\
\%, \\
\text { Excelle } \\
\text { nt0\% }\end{array}$ & $\begin{array}{l}\text { Zero0 } \\
\%, \\
\text { Poor0 } \\
\%, \\
\text { Fair14 } \\
\%, \\
\text { Good8 } \\
6 \%, \\
\text { Very } \\
\text { Good0 } \\
\%, \\
\text { Excelle } \\
\text { nt0\% }\end{array}$ & van & $\begin{array}{l}\text { Zero0 } \\
\%, \\
\text { Poor0 } \\
\%, \\
\text { Fair14 } \\
\%, \\
\text { Good8 } \\
6 \%, \\
\text { Very } \\
\text { Good0 } \\
\%, \\
\text { Excelle } \\
\text { nt0\% }\end{array}$ & $\begin{array}{l}\text { Zero10 } \\
0 \%, \\
\text { Poor0 } \\
\%, \\
\text { Fair0\% } \\
\text { ' } \\
\text { Good0 } \\
\%, \\
\text { Very } \\
\text { Good0 } \\
\%, \\
\text { Excelle } \\
\text { nt0\% }\end{array}$ & $\begin{array}{l}\text { Zero0 } \\
\%, \\
\text { Poor0 } \\
\%, \\
\text { Fair0\% } \\
\text { Good0 } \\
\text { \%, } \\
\text { Very } \\
\text { Good1 } \\
\text { 00\%, } \\
\text { Excelle } \\
\text { nt0\% }\end{array}$ \\
\hline 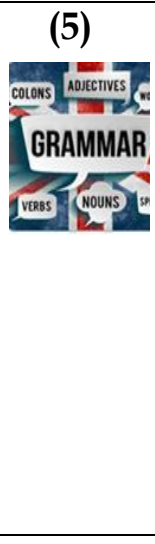 & $\begin{array}{l}\text { Zero } \\
0 \%, \\
\text { Poor } \\
0 \%, \\
\text { Fair } \\
28 \%, \\
\text { Good } \\
72 \%, \\
\text { Very } \\
\text { Good } \\
0 \%, \\
\end{array}$ & $\begin{array}{l}\text { Zero0 } \\
\%, \\
\text { Poor0 } \\
\%, \\
\text { Fair14 } \\
\%, \\
\text { Good8 } \\
6 \%, \\
\text { Very } \\
\text { Good0 } \\
\%,\end{array}$ & $\begin{array}{l}\text { Zero0 } \\
\%, \\
\text { Poor0 } \\
\%, \\
\text { Fair14 } \\
\%, \\
\text { Good8 } \\
6 \%, \\
\text { Very } \\
\text { Good0 } \\
\%,\end{array}$ & $\begin{array}{l}\text { Zero0 } \\
\%, \\
\text { Poor0 } \\
\%, \\
\text { Fair14 } \\
\%, \\
\text { Good7 } \\
2 \%, \\
\text { Very } \\
\text { Good1 } \\
4 \%,\end{array}$ & levels & $\begin{array}{l}\text { Zero0 } \\
\%, \\
\text { Poor0 } \\
\%, \\
\text { Fair14 } \\
\%, \\
\text { Good8 } \\
6 \%, \\
\text { Very } \\
\text { Good0 } \\
\%,\end{array}$ & $\begin{array}{l}\text { Zero0 } \\
\%, \\
\text { Poor28 } \\
\%, \\
\text { Fair72 } \\
\%, \\
\text { Good0 } \\
\%, \\
\text { Very } \\
\text { Good0 } \\
\%,\end{array}$ & $\begin{array}{l}\text { Zero0 } \\
\%, \\
\text { Poor0 } \\
\%, \\
\text { Fair0\% } \\
\text { ' } \\
\text { Good0 } \\
\%, \\
\text { Very } \\
\text { Good1 } \\
00 \%, \\
\end{array}$ \\
\hline
\end{tabular}




\begin{tabular}{lllllll}
\hline Excelle & Excelle & Excelle & Excelle & Excelle & Excelle & Excelle \\
nt $0 \%$ & nt0\% & nt0\% & nt0\% & nt0\% & nt0\% & nt0\%
\end{tabular}

\begin{tabular}{|c|c|c|c|c|c|c|c|c|}
\hline (6) & $\begin{array}{l}\text { Zero0 } \\
\%\end{array}$ & $\begin{array}{l}\text { Zero0 } \\
\%\end{array}$ & $\begin{array}{l}\text { Zero0 } \\
\%\end{array}$ & $\begin{array}{l}\text { Zero0 } \\
\%\end{array}$ & $\begin{array}{l}\text { elementa } \\
\text { ry }\end{array}$ & $\begin{array}{l}\text { Zero0 } \\
\%\end{array}$ & $\begin{array}{l}\text { Zero0 } \\
\%\end{array}$ & $\begin{array}{l}\text { Zero0 } \\
\%\end{array}$ \\
\hline & Poor0 & Poor0 & Poor0 & Poor0 & & Poor0 & Poor14 & Poor0 \\
\hline & $\%$ & $\%$, & & $\%$, & & & & \\
\hline & Fair14 & $\begin{array}{l}\text { Fair14 } \\
\%,\end{array}$ & $\begin{array}{l}\text { Fair14 } \\
\%,\end{array}$ & $\begin{array}{l}\text { Fair14 } \\
\%\end{array}$ & & $\begin{array}{l}\text { Fair14 } \\
\%\end{array}$ & $\begin{array}{l}\text { Fair72 } \\
\%\end{array}$ & Fair42 \\
\hline & & Good8 & Good8 & Good8 & & Good7 & Good1 & \\
\hline & Good8 & $6 \%$, & $6 \%$, & $6 \%$, & & $2 \%$, & $4 \%$, & Good5 \\
\hline & 6\%, & Very & Very & Very & & Very & Very & $8 \%$ \\
\hline & Very & Good0 & Good0 & Good0 & & Good1 & Good0 & Very \\
\hline & Good0 & $\%$, & $\%$, & $\%$, & & $4 \%$, & $\%$, & Good0 \\
\hline & $\%$, & Excelle & Excelle & Excelle & & Excelle & Excelle & \\
\hline & $\begin{array}{l}\text { Excelle } \\
\text { nt } 0 \%\end{array}$ & nt0\% & nt0\% & nt0\% & & nt0\% & nt0\% & $\begin{array}{l}\text { Excelle } \\
\text { nt } 0 \%\end{array}$ \\
\hline
\end{tabular}

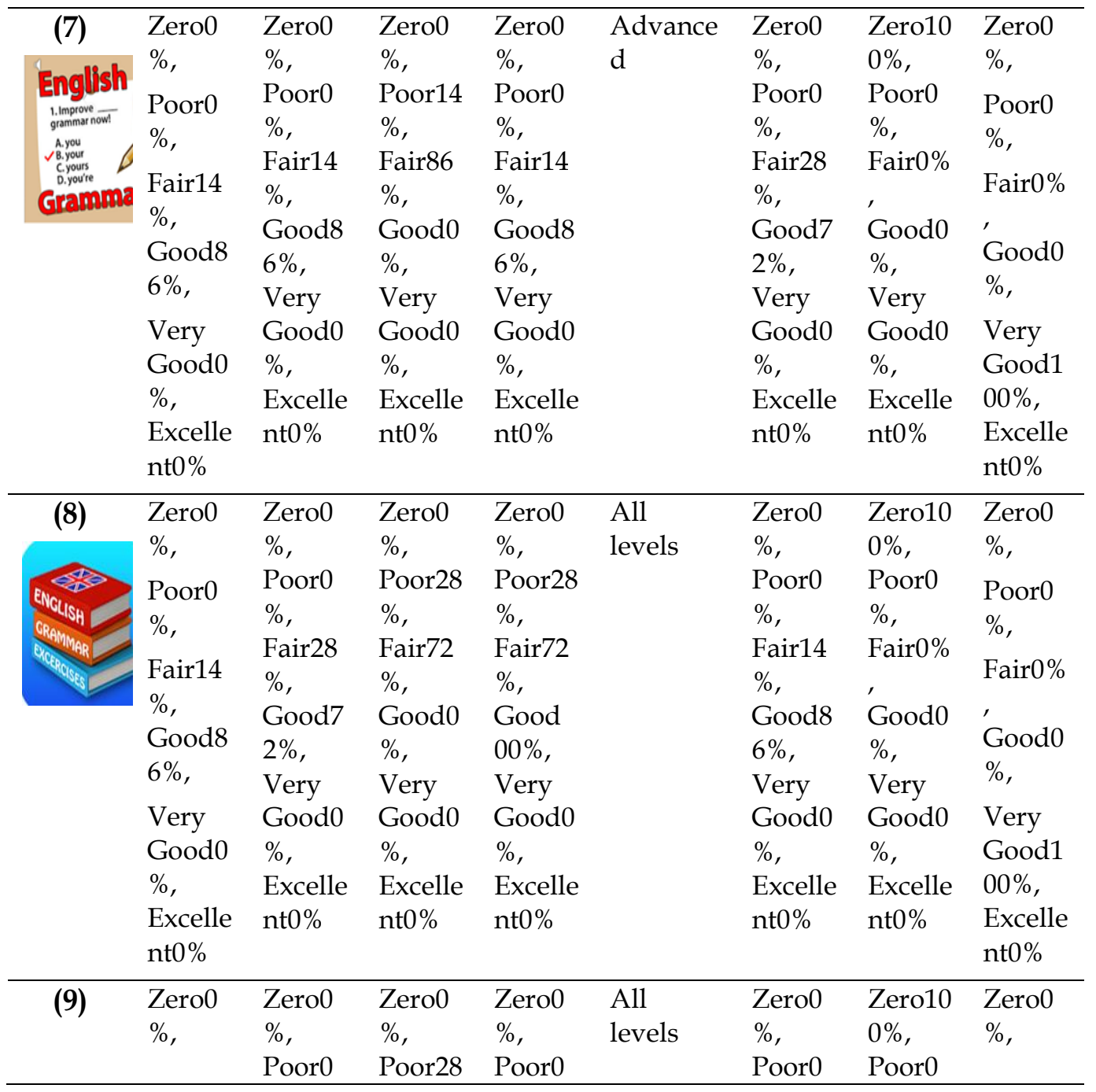




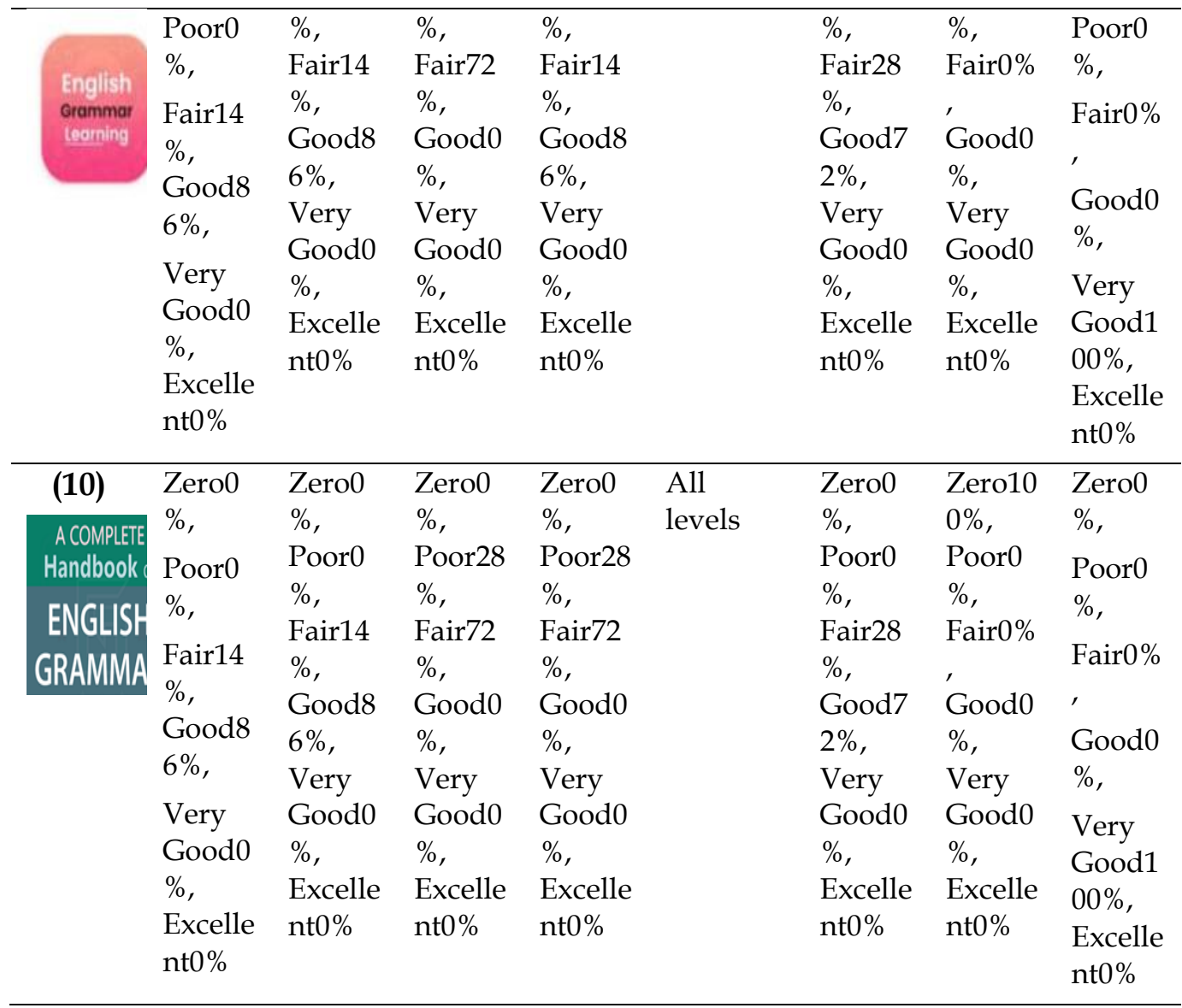

As indicated in Table 4 above, the features of apps connected with curriculum and language \& culture are analyzed.

In reference to curriculum, evaluators have rated app 1(Poor14\%, Fair86\%, Good0\%), app2 (Fair14\%, Good86\%), app3 (Good28\%, Very Good72\%), app4 (Fair14\%, Good72\%, Very Good14\%), app5 (Fair28\%, Good72\%), app6 (Fair14\%, Good86\%), app7(Fair14\%, Good86\%),app8 (Fair14\%, Good86\%), app9 (Fair14\%, Good $86 \%$ ), and app10(Fair14\%, Good86\%). The finding indicates that 9 apps have quite good learning sequence, while pps3 has very good learning sequence.

Another feature of curriculum is skill development. Evaluators have rated app 1(Fair14\%, Good86\%), app2 (Fair28\%, Good72\%), app3(Fair14\%, Good86\%), app4(Fair14\%, Good86\%), app5 (Fair14\%, Good86\%), app6(Fair14\%, Good86\%), app7 (Fair14\%, Good86\%), app8 (Fair28\%, Good72\%), app9 (Fair14\%, Good86\%), and app10 (Fair $14 \%$, Good $86 \%$ ). The findings show that almost all apps have quite good skill development feature.

Another feature related to curriculum is MALL role. Evaluators have rated app 1(Fair14\%， Good86\%), app 2(Fair14\%， Good86\%), app3(Good100\%), app4(Poor14\%, Fair72\%, Good14\%), app5(Fair14\%, Good86\%), app6 (Fair14\%, Good86\%), app7 (Poor14\%, Fair86\%), app8 (Poor28\%, Fair72\%), app9 (Poor28\%, Fair72\%), and app10 (Poor28\%, Fair72\%). It was found that five apps (1, 2, 3, 5\&6) have quite good MALL role, while the rest $(4,7,8,9, \& 10)$ has a quite fair MALL role.

Another feature associated with curriculum is autonomous. Evaluators have rated app1 (Fair14\%, Good86\%), app2 (Fair14\%, Good72\%, Very Good14\%), app3 
(Fair14\%, Good72\%, Very Good14\%), app4 (Fair14\%, Good86\%), app5 (Fair14\%, Good72\%, Very Good14\%), app6 (Fair14\%, Good86\%), app7 (Fair14\%, Good86\%), app8 (Poor28\%, Fair72\%), app9 (Fair14\%, Good86\%), and app10 (Poor28\%, Fair72\%, Good0\%). The study found that 8 apps have quite good autonomy for language learning, while two apps $(8 \& 10)$ have quite fair language learning autonomy. Another feature related to curriculum is proficiency level. Evaluators indicated that 7 apps can be used for users of all language ability level, while apps 6,4 , and 7 are suitable for elementary, Inter/advanced and advanced levels, respectively.

In relation to complexity level of language and culture, evaluators have rated app 1(Fair14\%, Good72\%, Very Good14\%), app2 (Fair28\%, Good72\%), app3 (Good28\%, Very Good72\%), app4 (Fair14\%, Good86\%), app5 (Fair14\%, Good86\%), app6 (Fair14\%, Good72\%, Very Good14\%), app7 (Fair28\%, Good72\%), app8 (Fair14\%, Good86\%), app9 (Fair28\%, Good72\%, Very), and app10 (Fair28\%, Good $72 \%)$. The findings showed that overall the apps have quite good complexity level.

Another feature related to language and culture is cultural element. The evaluators have rated app1 (Zero100\%), app2 (Fair28\%, Good 72\%), app3(Poor28\%, Fair 72\%), app4 (Zero100\%), app5 (Poor28\%, Fair72\%), app6 (Poor14\%, Fair72\%, Good14\%), and app7, 8, 9, and 10 equally as (Zero 100\%). One app has a quite good cultural element embedded, while two apps (3\&5) have been rated as fair in terms of cultural element; however, six apps $(1,4,7,8,9, \& 10)$ have no cultural elements.

The last feature related to language and culture is spelling \& grammar accuracy. Evaluators have rated app 1(Very Good 100\%), app2 (Good 100\%), app3 (Good 100\%), app4 (Very Good 100\%), app5 (Very Good 100\%), app6 (Fair42\%, Good $58 \%$ ), apps7,8,9, and 10 equally (Very Good $100 \%)$. Data analysis revealed that 7 apps have been rated as 'very good' in terms of spelling and accuracy. Two apps (2\&3) were selected as good, while one app (6) was selected to be with the least accuracy.

\section{Discussion}

As indicated in Table 4 above, the features of apps connected with curriculum and language \& culture are analyzed.

In reference to curriculum, evaluators have rated app 1(Poor14\%, Fair86\%, Good0\%), app2 (Fair14\%, Good86\%), app3 (Good28\%, Very Good72\%), app4 (Fair14\%, Good72\%, Very Good14\%), app5 (Fair28\%, Good72\%), app6 (Fair14\%, Good86\%), app7(Fair14\%, Good86\%),app8 (Fair14\%, Good86\%), app9 (Fair14\%, Good $86 \%$ ), and app10(Fair14\%, Good86\%). The finding indicates that 9 apps have quite good learning sequence, while pps3 has very good learning sequence.

Another feature of curriculum is skill development. Evaluators have rated app 1(Fair14\%, Good86\%), app2 (Fair28\%, Good72\%), app3(Fair14\%, Good86\%), app4(Fair14\%, Good86\%), app5 (Fair14\%, Good86\%), app6(Fair14\%, Good86\%), app7 (Fair14\%, Good86\%), app8 (Fair28\%, Good72\%), app9 (Fair14\%, Good86\%), and app10 (Fair14\%, Good $86 \%$ ). The findings show that almost all apps have quite good skill development feature. 
Another feature related to curriculum is MALL role. Evaluators have rated app 1(Fair14\%, Good86\%), app 2(Fair14\%, Good86\%), app3(Good100\%), app4(Poor14\%, Fair72\%, Good14\%), app5(Fair14\%, Good86\%), app6 (Fair14\%, Good86\%), app7 (Poor14\%, Fair86\%), app8 (Poor28\%, Fair72\%), app9 (Poor28\%, Fair72\%), and app10 (Poor28\%, Fair72\%). It was found that five apps $(1,2,3,5 \& 6)$ have quite good MALL role, while the rest $(4,7,8,9, \& 10)$ has a quite fair MALL role.

Another feature associated with curriculum is autonomous. Evaluators have rated app1(Fair14\%, Good86\%), app2(Fair14\%, Good72\%, Very Good14\%), app3(Fair14\%, Good72\%, Very Good14\%), app4(Fair14\%, Good86\%), app5(Fair14\%, Good72\%, Very Good14\%), app6 (Fair14\%, Good86\%), app7(Fair14\%, Good86\%), app8 (Poor28\%, Fair72\%), app9(Fair14\%, Good86\%), and app10(Poor $28 \%$, Fair72\%, Good0\%). The study found that 8 apps have quite good autonomy for language learning, while two apps $(8 \& 10)$ have quite fair language learning autonomy. Another feature related to curriculum is proficiency level. Evaluators indicated that 7 apps can be used for users of all language ability level, while apps 6,4, and 7 are suitable for elementary, Inter/advanced and advanced levels, respectively.

In relation to complexity level of language and culture, evaluators have rated app 1(Fair14\%, Good72\%, Very Good14\%), app2 (Fair28\%, Good72\%), app3 (Good28\%, Very Good72\%), app4 (Fair14\%, Good86\%), app5 (Fair14\%, Good86\%), app6 (Fair14\%, Good72\%, Very Good14\%), app7 (Fair28\%, Good72\%), app8 (Fair14\%, Good86\%), app9 (Fair28\%, Good72\%, Very), and app10 (Fair28\%, Good $72 \%)$. The findings showed that overall the apps have quite good complexity level.

Another feature related to language and culture is cultural element. The evaluators have rated app1 (Zero100\%), app2 (Fair28\%, Good72\%), app3(Poor28\%, Fair72\%), app4(Zero100\%), app5 (Poor28\%, Fair72\%), app6 (Poor14\%, Fair72\%, Good14\%), and app7, 8, 9, and 10 equally as (Zero100\%).One app has a quite good cultural element embedded, while two apps (3\&5) have been rated as fair in terms of cultural element; however, six apps $(1,4,7,8,9, \& 10)$ have no cultural elements.

The last feature related to language and culture is spelling \& grammar accuracy. Evaluators have rated app 1(Very Good100\%), app2 (Good100\%), app3 (Good100\%), app4 (Very Good100\%), app5 (Very Good100\%), app6 (Fair42\%, Good58\%), apps7,8,9, and 10 equally (Very Good100\%). Data analysis revealed that 7 apps have been rated as 'very good' in terms of spelling and accuracy. Two apps (2\&3) were selected as good, while one app (6) was selected to be with the least accuracy.

\section{Implication}

The findings of the current study have implications for students, lectures, curriculum designers, and MALL app designers. The students are introduced to some applications which are useful for autonomous, selfmanaged, self-directed, and self-paced learning, where they can appreciate 
informal, ubiquitous, spontaneous, personalized learning process (Miangah \& Nezarat 2012) reflecting the feature of MALL.

The lecturers may take advantage of the findings of this study to supplement their teaching language in general, and grammar in particular. It may expedite the use of flipped classroom and blended learning using online technology. As the education paradigm has shifted from face-to face to online teaching and learning, teachers should take advantage of online apps; however, they should select for or guide their students in choosing the right apps for learning language. Moreover, students as language app users should be critical in the selection of apps for autonomous language learning. They must be aware of the fact that not all language apps are valid or reliable to use when learning language.

The findings have also implications for curriculum designers in designing, and selecting appropriate language learning materials catering to the needs, interests, styles and preferences of language learners particularly in the view of pandemic as the education is done online. Curriculum designers must also be aware of those language apps developed by nonprofessionals. The finding also implies that the MALL app developers should take some factors into considerations, such as: adverts and bug, accuracy of app content in terms of grammar and spelling, embedding principles of behaviorism (e.g., drill and practice), cognitivism (e.g., problem solution), constructivism (problem solution, collaborative learning), and connectivism (networking and self-organization). They also need to focus more on MALL activities both conceptually and technically. In addition, the app developers might be suggested that they alleviate the technical issues of the apps which could slow down their usage.

\section{Conclusion}

This paper has analyzed 10 currently openly available mobile language learning apps for grammar learning. The freely available apps were selected based on the high ranking given by the users ( 3.7 and above out of 5 ) and the high number of installation. The findings show that all apps have advertisement which could sometimes be irritating and tedious. The apps have issues in simulation and problem-solving activities. Their accuracy (in terms of grammar and spelling) and cultural element need to be promoted. Some apps have no testing and assessment system and they mostly lack explanatory feedback. Some apps have inaccuracy issue in terms of grammar and spelling. The MALL role needs to be enhanced in some apps as it is far from satisfactory. The apps tend not to be pedagogically and technically go hand in hand with language learning and grammar improvement satisfactorily. Overall, the apps need to be improved in terms of system, program, curriculum and language \& culture.

The present study has attempted to analyze the ten selected grammar apps comprehensively; however, it has some limitations which need to be covered in future studies to gain a full picture in evaluation and assessment of mobile 
language learning apps. It might be of interest to look at students' perceptions of and attitudes towards the apps for grammar learning and improvements through survey and interview. It goes without saying that, an experimental study with experiment and control groups is of interest to see the real effect of using mobile apps for language learning and grammar improvement in particular. More ample sampling of apps is needed to reach a better conclusion though the current number of apps is a sound start in this direction. Further, observation of students' use of apps could also be a future prospective research as it may highlight the issues and challenges of using apps. Lastly, instructors' voices regarding app functions and features might be explored through interview.

\section{References}

Brown, H. D. (2007). Teaching by principles: An interactive approach to language pedagogy. White Plains, NY: Pearson Education.

Blyth, C. (2007). A constructivist approach to grammar: Teaching teachers to teach aspect. The Modern Language Journal, 81(1), 50-66. Retrieved from http://www.jstor.org/stable/329160.

Chen, X. (2016). Evaluating language-learning mobile apps for second-language learners. Journal of Educational Technology Development and Exchange (JETDE), 9(2), 39-51.

Creswell, J. W. (2014). A concise introduction to mixed methods research. Sage Publications.

Deng, Q \&Trainin, G. (2015). Learning Vocabulary with Apps: From Theory to Practice. The Nebraska Educator. A Student-Led Journal. 29,49-69.

Eppard, J., Nasser, O., \& Reddy, P. (2016). The next generation of technology: Mobile apps in the English language classroom. International Journal of Emerging Technologies in Learning (iJET), 11(4), 21-27.

Fabian, K., \& MacLean, D. (2014). Keep taking the tablets? Assessing the use of tablet devices in learning and teaching activities in the Further Education sector. Research in Learning Technology, 22.

Fosnot, C. T., \& Perry, R. S. (1996). Constructivism: A psychological theory of learning. Constructivism: Theory, perspectives, and practice, 8-33.

Ganapathy, M., Shuib, M., \& Azizan, S. N. (2016). Malaysian ESL students' perceptions on the usability of a mobile application for grammar test: A case study of ESL undergraduates in UniversitiSains Malaysia. 3L. Language, Linguistics, Literature, 22(1), 127-140.

Google Play Store. Retrieved from https:// play.google.com/store. July 2021

Heil, C. R., Wu, J. S., Lee, J. J., \& Schmidt, T. (2016). A review of mobile language learning applications: Trends, challenges, and opportunities. The EuroCALL Review, 24(2), 32-50.

Kim, H., \& Kwon, Y. (2012). Exploring smartphone applications for effective mobile-assisted language learning. Multimedia-Assisted Language Learning, 15(1), 31-57. 
Kukulska-Hulme, A., \& Shield, L. (2009). An overview of mobile assisted language learning: From content delivery to supported collaboration and interaction. ReCALL, 20(3), 271-289.

Kukulska-Hulme, A., Pettit, J., Bradley, L., Carvalho, A., Herrington, A., Kennedy, D., \& Walker, A. (2011).Mature Students Using Mobile Devices in Life and Learning. International Journal of Mobile and Blended Learning, 31(1), 1852.

Kukulska-Hulme, A., \& Bull, S. (2009). Theory-based support for mobile language learning: Noticing and recording. International Journal of Interactive Mobile Technologies, 3, 12-18.

León, A. M., Bravo, C. B., \& Fernández, A. R. (2017). Review of Android and iOS tablet apps in Spanish to improve reading and writing skills of children with dyslexia. Procedia-Social and Behavioral Sciences, 237, 1383-1389.

Miangah, T. M., \&Nezarat, A. (2012). Mobile-assisted language learning. International Journal of Distributed and Parallel Systems, 3(1), 309.

Martín-Monje, E., Arús, J., Rodríguez-Arancón, P., \& Calle-Martínez, C. (2014). REALL: Rubric for the evaluation of apps in language learning.

Ormrod, J. E., \& Davis, K. M. (2004). Human learning. London: Merrill.

Oxford, R. L. (1995). Linking theories of learning with intelligent computerassisted language learning (ICALL). In V.M. Holland, M.R. Sams, \& J.D. Kaplan (Eds.) Intelligent language tutors: Theory shaping technology (359-369). Lawrence Erlbaum Associates, Inc.: Mahwah, New Jersey.

Pilar, R. A., Jorge, A., \& Cristina, C. (2013). The use of current mobile learning applications in EFL. Procedia-Social and Behavioral Sciences, 103, 11891196.

Reinders, H., \& Pegrum, M. (2016). Supporting Language Learning on the Move: An Evaluative Framework for Mobile Language Learning Resources. In B. Tomlinson (Ed.), Research and Materials Development for Language Learning (pp. 219-232). NewYork,NY: Routledge.

Rodríguez-Arancón, P., Arús, J., \& Calle-Martínez, C. (2013). The use of current mobile learning applications in EFL.

Siemens, G. (2008). Learning and knowing in networks: Changing roles for educators and designers. ITFORUM for Discussion, 27(1), 1-26.

Sung, Y. T., Chang, K. E., \& Liu, T. C. (2016). The effects of integrating mobile devices with teaching and learning on students' learning performance: A meta-analysis and research synthesis. Computers \& Education, 94, 252-275.

Supyan Hussin (2013). Mobile Apps for mobile Assisted language leaning Good or Bad? Seminar Interactive Mobile for Learning and $7^{\text {th }}$ international malaysian educational technology ITMET Convention, Universiti Kebangsaan Malaysia.

Usagawa, T. (2018). Investigation of Students' Mobile Phone Usage and Influences towards Their Mobile Learning Adoption: A Case Study in Myanmar. International Journal of Interactive Mobile Technologies (iJIM), 12(5), 43-57. 
Wang W, Li Y, Wang X, Liu J, Zhang X (2017). Detecting android malicious apps and categorizing benign apps with ensemble of classifiers. Future Generation Computer Systems. Available: http://dx.doi.org/10.1016/j.future.2017.01.019.

Wu, W. H., Wu, Y. C. J., Chen, C. Y., Kao, H. Y., Lin, C. H., \& Huang, S. H. (2012). Review of trends from mobile learning studies: A meta-analysis. Computers E Education, 59(2), 817-827.

Yunita, A., Nursechafia, N., Setiawan, E., Nugroho, H., \& Ramadhan, H. (2018). The Relationship between Mobile Phone Usage in Classroom and Academic Achievement in College Life. International Journal of Interactive Mobile Technologies (IJIM), 12(8),96-103. 